\title{
The Desire to Die in Palliative Care: A sequential mixed methods study to develop a semi-structured clinical guideline
}

\author{
Kerstin Kremeike ( $\nabla$ kerstin.kremeike@uk-koeln.de) \\ Uniklinik Köln, Zentrum für Palliativmedizin https://orcid.org/0000-0003-4316-2379 \\ Gerrit Frerich \\ University of Cologne, Medical Faculty, Departement of Palliative Medicine
}

Vanessa Romotzky

University of Cologne, Medical Faculty, Departement of Palliative Medicine

\section{Kathleen Bostroem}

University of Cologne, Medical Faculty, Departement of Palliative Medicine

Thomas Dojan

University of Cologne, Medical Faculty, Departement of Palliative Medicine

Maren Galushko

Uniklinik Köln, Zentrum für Palliativmedizin

Kija Shah-Hosseini

Institute of Medical Sociology, Health Services Research, and Rehabilitation Science (IMVR), University of Cologne

Saskia Juenger

Cologne Center for Ethics, Rights, Economics, and Social Sciences of Health (ceres), University Clinic of Cologne

Gary Rodin

Departement of Supportive Care, Princess Margret Cancer Centre, University Health Network Toronto

Holger Pfaff

Institute of Medical Sociology, Health Services Research, and Rehabilitation Science (IMVR), University of Cologne

Klaus Maria Perrar

University of Cologne, Medical Faculty, Departement of Palliative Medicine

Raymond Voltz

University of Cologne, Medical Faculty, Departement of Palliative Medicine

\section{Research article}

Keywords: desire to die, wish towards hastened death, suicidal ideation, relationship, communication, palliative care, professionals, consensus, patients, Germany

Posted Date: January 14th, 2020

DOI: https://doi.org/10.21203/rs.2.20851/v1

License: (1) This work is licensed under a Creative Commons Attribution 4.0 International License. Read Full License

Version of Record: A version of this preprint was published at BMC Palliative Care on April 16th, 2020. See the published version at https://doi.org/10.1186/s12904-020-00548-7. 


\section{Abstract}

Objective: Although desire to die (DD) of varying intensity and permanence is frequent in patients with advanced cancer and those receiving palliative care, uncertainty exists concerning appropriate therapeutic responses to it. To support health professionals in dealing with patients' potential DD, a training program and a semi-structured clinical interview guideline was developed. This study aimed for a revision of and consensus building on the clinical guideline to support proactively addressing DD and routine exploration of death and dying distress.

Methods: Within a sequential mixed methods design, we invited 16 palliative patients to participate in semi-structured interviews and 377 (inter-)national experts to attend a two-round Delphi process. Interviews were analyzed using qualitative content analysis and an agreement consensus for the Delphi was determined according to predefined criteria.

Results : 11 (69\%) patients from different settings participated in face-to-face interviews. As key issues for conversations on DD they pointed out the relationship between professionals and patients, the setting and support from external experts, if required. A set of 149 (40\%) experts (132/89\% from Germany, 17/11\% from 9 other countries) evaluated ten domains of the clinical guideline. There was immediate consensus on nine domains concerning conversation design, suggestions for (self-)reflection, and further recommended action. The one domain in which consensus was not achieved until the second round was "proactively addressing DD".

Conclusions: We have provided the first semi-structured clinical guideline to identify and address DD and to respond therapeutically - based on evidence, patients' views and consensus among (inter-)national professional experts. Trial registration: German Clinical Trials Register (No. DRKS00012988).

\section{Background}

Desire to Die (DD) is a complex phenomenon with individual reasons, forms and consequences. ${ }^{1,2}$ We use the term DD in a broad sense including an acceptance of death, a wish for hastened death (WTHD) without requiring any accelerating action, ${ }^{3}$ a request for assisted dying and suicidal ideation. ${ }^{4}$ This understanding of DD differs from the international consensus definition of the WTHD ${ }^{5}$ and refers to the German PC guideline for patients with incurable cancer. ${ }^{6}$ The broad definition is meant to foster a more open communication with patients as it supports HPs' acceptance of DD as a potential way to cope with a terminal illness.

DD can coexist with a simultaneous will to live ${ }^{7}$, with both prone to change over time. ${ }^{1,8}$ DD is connected with physical and psychological distress ${ }^{9}$ and can be the beginning of a suicidal process, ${ }^{10}$ but studies have shown that communication concerning therapeutic options may ease patient's burden, and even prevent suicides. ${ }^{11}$

Some forms of DD are frequent in patients in their last months of life. In a 1995 study, $45 \%$ of 200 advanced cancer patients showed at least occasional DD and almost $10 \%$ reported a strong and persistent one. ${ }^{12} \mathrm{~A}$ recent survey of 377 cancer patients found that $18 \%$ reported an occasional DD and $12 \%$ a serious one. ${ }^{13}$ Although health professionals (HPs) are frequently confronted with a patient's $\mathrm{DD},{ }^{3}$ it is not routinely assessed in palliative care (PC). A lack of preparation on how to deal with the complex and sensitive topic contributes to uncertainty in HPs about how to approach DD in clinical practice. ${ }^{14}$ This could lead them to neglect or insufficiently discuss the issue ${ }^{14}$ even if raised by the patient.

Current recommendations suggest proactively addressing $D D,{ }^{6}$ referencing studies which found positive effects on patients. ${ }^{8,15}$ These effects include opening communication about emotional conditions, even in absence of DD. ${ }^{16}$ Additionally, DD may also be assessed by validated instruments such as the Schedules of Attitudes for Hastened Death (SAHD; primary for research purposes) ${ }^{8}$ or the Desire for Death Rating Scale (DDRS; initially developed for clinical interviews). ${ }^{17}$

Training programs and recommendations for talking about difficult issues ${ }^{18,19}$ may also support HPs in dealing with DD. ${ }^{20} \mathrm{~A}$ training program regarding DD has been developed in a previous project, based on a literature review and results of focus groups with multiprofessional German PC providers. ${ }^{21}$ This training program has been piloted and evaluated through a 32 -item-scale covering the dimensions of self-confidence, skills, knowledge and attitudes. Descriptive changes indicate a major improvement in self-confidence and at least minor improvements on all dimensions after three months, with only one item concerning knowledge reaching statistical 
significance, though. ${ }^{21}$ Within the development of the training program, a clinical guideline was drafted, building on published recommendations on dealing with $\mathrm{DD}^{22,23}$ and based on results from an interdisciplinary advisory board discussion. The draft was refined and structured within a framework analysis approach. ${ }^{21}$ The present study aimed at further developing and refining the clinical guideline for routinely assessing death and dying distress, reacting to and (proactively) addressing DD - taking into account international expertise from a wider range of professions, patient representatives and relatives as well as the voices of patients.

\section{Methods}

A sequential mixed methods design was used including qualitative patient interviews and a Delphi survey with experts (see Fig. 1). Both methods were chosen to maximize the guideline's clinical relevance. More details on the background on and justification of the methods selected are described in the study protocol. ${ }^{24}$ We applied the consolidated criteria for reporting qualitative research (COREQ) ${ }^{25}$ and the guidelines for conducting and reporting Delphi Studies (CREDES) in PC. ${ }^{26}$ Research was conducted according to the Declaration of Helsinki. Ethical approval for this study was obtained from the Ethics Committee of the University of Cologne (\#17-265).

\section{Patient interviews}

Patient interviews were conducted to consider the perspective of palliative patients and thereby strengthen the appropriateness of the guideline. A convenience sample of 16 adult patients receiving PC was invited to participate in face-to-face interviews on the appropriate approach to DD in PC. Participants were recruited via PC providers known to the research team. A leaflet for patients and HPs included survey aims, procedure and contact details of the research team. HPs contacted the researchers in case of patients' willingness to participate. The research team approached these patients to obtain informed consent for study participation.

Interviewees chose the location of the interview, which were conducted by either one of three female (KK, VR, KB) or one male (GF) interviewers. Two researchers hold doctoral degrees (KK, GF), one was a doctoral student (VR), the other a graduate student (KB). At the time of data acquisition, all researchers worked for the Department of Palliative Medicine at the University Hospital of Cologne and were trained and experienced in conducting semi-structured interviews. This approach to data acquisition allowed the research agenda to be pre-defined to some extent and at the same time enabled respondents to freely present a range of views and new insights. ${ }^{27}$ The interview guide was developed by a group of social scientists (KK, GF, MG), psychologists (TD, KB), a pedagogue (VR) and a physician (KMP). It assessed aspects relevant to addressing DD, desirable traits in interviewer as well as interviewees' potential personal DD. The complete interview guideline is added as an additional file (see Additional file 1). Social demographics were assessed using a brief questionnaire.

\section{Delphi survey}

In addition to patient interviews, an online Delphi survey was performed to achieve expert consensus on content and structure of the clinical guideline. We invited participants from 13 countries aiming to compose a balanced sample of 50-70 researchers and clinical practitioners. Recruitment took place through personal contacts and an internet search. Potential participants were asked via email to take part in the survey and to propose further experts. As nurses were initially underrepresented in recruitment, we reached out to the nursing mailing list of the German Society for Palliative Medicine, which distributed our call to a selection of its members. This pushed the number of invited participants to 377 .

To develop the Delphi questionnaire, we revised the existing version of the clinical guideline ${ }^{21}$ based on our preceding patient interviews. The revised draft included three sections ( $B$ to $D$ in Table 2) with a total of ten domains each containing a set of suggestions for HPs to take into account when (proactively) discussing DD with palliative patients. The Delphi survey was designed accordingly and conducted in co-operation with an external partner (UZ Bonn, Society for Empirical Social Research and Evaluation).

Each panelist received a link to the survey via email. During both Delphi rounds two reminders were sent out to motivate nonrespondents to participate. During the first Delphi round, participants evaluated each domain of the guideline by a) rating the importance of the entire section presented on a five-point Likert scale ranging from 0 ("very unimportant") to 5 ("very important") and b) giving free text comments on the suggestions listed in the domain they just rated. In the second Delphi round, participants were asked to reevaluate the guideline in light of the results of the first round. Participants were also fed back information about the first rounds' sample seize such as distribution of professions and the sample's experience with DD and suicidality. 


\section{Data analysis}

Patient interviews were transcribed verbatim and analyzed by three researchers (KB, KK, GF) applying qualitative content analysis. ${ }^{28}$ Inductive and deductive categories were derived and applied using the qualitative data analysis software MAXQDA 12. Inter-coder reliability was ensured by constant comparison between coders and according adjustments throughout the entire process. Transcripts were not returned to participants and they were not asked to provide feedback on the findings.

Delphi panelists' data was eligible for analysis if (inclusion criteria): panelists were patient representatives, relatives or part of the relevant occupational group (multi-professional experts in the field, reported at least $\geq 5$ years of experience in dealing with DD and/or suicidality, and reached high scores in self-assessed confidence and knowledge with DD and/or suicidality (rating at least four on a 0 to 6 Likert scale on one of the four items)). Data from the Delphi survey was analyzed statistically for quantitative results using SPSS 23 (IBM Corp., Armonk, NY, USA). Thematic analysis was applied to analyze free text comments. An a priori criterion of $80 \%$ agreement among panelists was defined to determine consensus ${ }^{26}$ on the importance of a section, requiring a rating of at least four on a one to five Likert scale.

Further details on sampling, data collection and analysis are reported in the study protocol. ${ }^{24}$

\section{Results}

\section{Patient interviews}

Between 9/2017 and 1/2018, 11 (69\%) patients receiving PC participated in face-to-face interviews lasting nine to 79 minutes. Reasons for patients refusing to partake or HPs judging the patient incapable of participation were (acute) deterioration of physical $(3 / 5)$ or mental $(2 / 5)$ health status. Table 1 presents further details on interviewees.

All but one of the interviewees reported DD either as a wish for hastened death or as acceptance of death without requiring to hasten it. A desire for physician assisted suicide was reported explicitly once, and two interviewees preferred death to deteriorating symptoms. Isolation, the feeling of being a burden, hopelessness and fear of pain were reasons reported for DD.

Deductive categories based on topics from the interview guideline were complemented with inductive coding of the interviews, producing five main categories with various sub-categories: "Actively building the relationship", "Conversation partner", "Conversation context and structure", "Conversation set up and framework" and "Own Desire to Die ". Within the formulated categories, patients mainly confirmed the dimensions and recommendations of the clinical guideline draft, yet emphasized individuality:

Well, you can't develop a very RIGID guideline, I think. It'Il need to cover a vast spectrum, starting from one point at the bottom and spreading VERY, VERY wide apart at the top. (51-year-old male with colon cancer)

However, some sub-categories added valuable insight and contributed to the revision of the guideline draft. Those sub-categories are reported below.

Almost all of the interviewees appreciated the proactive assessment of DD by HPs:

Interviewer: Would it have been helpful to you, if [the HP] had addressed [the DD]?

Patient: Yes. I think so. [...] I don't know how other patients feel, but talking about it was very difficult for me. (63-year-old female with abdominal cancer)

To initiate and discuss problems, establishing a trustful health-professional-patient-relationship is a prerequisite, which both sides have to allow for. Unobtrusively signaling an appreciative attitude best frames the setting: conscious eye contact and relational touch can help to establish intimacy, if appropriate:

Patient: Who among you [HPs] even gives hugs anymore or takes someone's arm? [...] You all have a hard time with that. Interviewer: This would be something important to you? 
Patient: Of COURSE. (57-year-old male with lower jaw cancer)

When arranging for an appropriate environment to talk about DD, privacy and the patient's mental state should be taken into account. Furthermore, taking enough time was unanimously appreciated and considered helpful in signaling special attention.

One patient reported that thoughts concerning hastened death primarily arose upon first confrontation with her cancer diagnosis. Breaking bad news was also experienced as traumatic by other cancer patients.

While most of the patients preferred to talk to their physician, all members of the PC team were deemed fit for a dialogue about DD. If required, participants indicated that support from external experts should be sought:

Patient: [The HP said:] "We can call a pastoral worker for you [...] to talk to." [...] I didn't KNOW what to talk about with a pastoral worker. But he was here for an hour and there WAS a lot to talk about, apparently. (63-year-old female with uterine cancer)

\section{Delphi survey}

Based on the clinical guideline draft revised after the patient interviews, a Delphi survey was conducted between 1/2018 and 3/2018. Round one was open for 22 days; round two for 16 days. There was almost no dropout (5.0\%) between rounds; during the first round 210 invitees participated and 200 during round two. We excluded 61/51 panelists that did not meet inclusion criteria from analysis. Therefore, in both rounds, 149 participants were fit for data analysis according to inclusion criteria. This number goes far beyond our original recruitment plans, encompassing 50-70 panelists and is due to the overwhelming feedback from German (nursing) experts. With $91.3 \%$ of the sample reporting to work in direct patient contact, it consists largely of practitioners. For socio-demographic details on the Delphi sample see Table 1.

\section{Suitability and usefulness}

$87 \%$ of the respondents $(n=129)$ valued the structure of the clinical guideline as '(very) suitable', $95 \%(n=141)$ rated the clinical guideline to be '(very) useful' for everyday clinical practice.

\section{Importance of individual domains}

Table 3 displays the results of the importance ratings for Delphi round one and two.

For all domains except 'proactively addressing DD', the a priori consensus criterion ( $\geq 80 \%$ agreement) was met during the first Delphi round (see Table 2). As only four domains did not reach $90 \%$ of agreement, we took reaching $<90 \%$ to indicate potential for optimization and therefore asked our panelists to reevaluate all such domains (proactively addressing, classification, functions, further recommended action) in the second round. During round two, all domains except 'proactively addressing DD' reached an agreement $\geq 90 \%$. As our panelists opposed too narrow prompts on how to address DD, we changed all interrogative clauses and prompts in the original draft into the instructions and circumscriptions found in the final version of the clinical guideline (see Additional File 2). Other modifications based on Delphi results are reported in Table 4.

No significant statistical differences in ratings for all domains were found among subsamples (e.g. gender, expertise, selfassessments).

\section{Controversial Aspects}

Aside from the relatively moderate agreement on 'proactively addressing DD' during the Delphi process, classifying DD as thoughts of terminating life prematurely, wish for assisted suicide or active euthanasia in the clinical guideline was also met with concern. In the final version of the guideline, these classifications were nonetheless included but go hand in hand with introductory information that points out the broadness of the DD phenomenon. The introductory information in Additional file 3 shows this in more detail.

\section{Discussion}




\section{(Proactively) addressing desire to die within trustful health professional- patient-relationships}

Since DD may be a potential way to cope with advanced disease at some point during the disease trajectory, ${ }^{30}$ exploring it and allowing its emotional expression in conversation may be beneficial to all patients. DD can be expressed in different ways and proactively addressing it may help to clarify reasons more openly and at an earlier stage. However, in clinical practice, DD is not yet routinely assessed by HPs and talking about it is associated with discomfort. ${ }^{20}$

Concerns about adverse effects of discussing DD, e.g. triggering suicidal thoughts in patients, are widespread ${ }^{20}$ and became apparent in our Delphi survey. Current studies confirm that asking about suicidality causes no harm but may reduce experienced burden and distress. ${ }^{31}$ Based on preliminary studies ${ }^{20,32}$ these findings can likely be extrapolated to PC. Currently, our clinical guideline is only available for HPs attending the related multi-professional training program. Within this training program, we present the aforementioned findings and the broad definition of DD prior to handing out the guideline. This is meant to alleviate concerns about iatrogenic risks of addressing DD.

A recent descriptive study employed an ad hoc semi-structured clinical interview for proactive assessment of a wish to hasten death among advanced cancer patients. ${ }^{32}$ Participants did not experience this as distressing, but considered it important regardless of whether they were personally affected. The patients we interviewed indicated that they appreciated the initiation of DD conversations by HPs. (Proactively) addressing the issue in this way can open communication about patients' emotions, even in absence of DD ${ }^{16}$, which may help to build a trusting relationship that can help to preserve the will to live $\mathrm{e}^{33}$ and perhaps diminish suicidality. The German National Ethics Advisory Board recommends suicide prevention programs to counteract requests for assisted suicide and the importance of communication is stressed for suicide prevention. ${ }^{34}$ These have been the reasons for including the recommendation that DD should be (proactively) addressed in the 2019 edition of the German PC guideline for patients with incurable cancer. ${ }^{6}$ Establishing and maintaining a relationship are essential in addressing patients' $\mathrm{DD}^{20}$ so that the establishment of a therapeutic relationship should precede conversations about DD. Therefore, the clinical guideline places a special focus on actively building relationships.

\section{Framing, timing and patient-attunement of communication}

Although patients do not object to enquiries about potential DD, even when they do not personally have one, ${ }^{32}$ the impact is likely to depend on how and when such conversations take place. Various guidelines have been developed for the improvement of HP-patientcommunication in PC settings, e.g. "NURSE - Naming, Understanding, Respecting, Supporting, Exploring" for difficult conversation tasks in oncology ${ }^{35}$ and "SPIKES - A Six-Step Protocol for Delivering Bad News" for breaking bad news. ${ }^{18}$ An evaluation of the SPIKES implementation found that privacy positively influence patients' rating of their knowledge gained and the experience of the amount of time devoted to it as being sufficient. ${ }^{36}$ This is consistent with our interview results that the framing of conversations is of particular importance. As receiving bad news was experienced as traumatic and a trigger for DD by some of our interviewees suffering from cancer, it may be appropriate to delay this discussion to follow-up conversations.

As has been found regarding breaking of bad news in oncology ${ }^{37}$ and in light of the feedback from our Delphi panelists, the clinical guideline is designed to promote a patient-centered approach, giving suggestions on how to address potential DD while avoiding checklist-type interrogations. ${ }^{38}$ As it is also stated in the guideline's usage notes, there is no need to address DD in the same way for all patients and not all thematic aspects need to be addressed in every conversation about DD,.

\section{Appropriate (therapeutic) responses towards desire to die}

The most general intervention advised with respect to every patient with DD is the initiation of an open communication about it. The clinical guideline suggests before considering any other action, HPs should aim to understand the patient's wish, its backgrounds, meanings and functions. As severe physical symptoms are also related to $D_{1}{ }^{39}$ optimal symptom control is especially important in patients expressing such desire. Discussion of the expected disease trajectory and options for treatment can also decrease anxiety. ${ }^{40}$ 
For patients who experience physical suffering as unbearable, sensitively pointing out that therapy withdrawal or palliative sedation until death are legal possibilities to alleviate suffering within the statutory framework in Germany. Its administration needs to be tailored towards the individual's needs and in sensitive coordination with relatives. ${ }^{6}$

Diagnostic clarification of depression is indispensable as it has been shown to predict ${ }^{39}$ and moderate ${ }^{41} \mathrm{DD}$. Suicidality with completed suicide at its most extreme can be a manifestation of DD, yet is not necessarily to be equated with it. Therapeutic interventions such as Meaning-Centered Therapy ${ }^{45}$ and a supportive expressive therapy named Managing Cancer and Living Meaningfully (CALM $)^{42}$ have been shown to alleviate depressive symptoms in patients with advanced cancer. ${ }^{43-45}$

\section{Controversy concerning assisted suicide and active euthanasia}

Our Delphi panelists expressed only moderate agreement for directly addressing wishes for euthanasia or assisted suicide. Since euthanasia ( $\$ 216$ national criminal code (StGB)) and assistance of suicide against payment ( $\$ 217$ StGB) are forbidden in Germany, participants may have preferred to avoid naming such wishes because their realization would be illegal. Another concern expressed by our Delphi panelists was that acknowledging DD could pressure patients into actively seeking hastened death. Both scenarios point towards an underlying belief that enquiring about DD will cause DD to emerge. This view can lead HPs to avoid talking about it. We emphasize the importance of a clear distinction between acknowledging and agreeing with or even endorsing and supporting DD. Therefore, the legal situation in Germany is duly addressed in the mandatory training course that introduces the clinical guideline as a practical tool.

\section{Self-protection and self-care}

In addition to recommendations aimed at improving the patients' well-being, protecting the well-being of HPs is also important. Conversations about DD can be enriching but might also cause emotional stress. ${ }^{46} \mathrm{It}$ is therefore important for HPs to develop a sensitive grasp of their own feelings and to protect themselves from emotional overload. ${ }^{46}$ In order to manage discussions of DD appropriately, HPs should also be aware of own values, norms and their personal stance concerning death wishes. Supervision, case meetings and everyday peer exchange can help to deal with stress or difficult situations. ${ }^{47}$

\section{Study Limitations}

As $64 \%$ of our interview partners were cancer patients the results appear applicable to oncological settings, but the generalizability of findings onto other palliative patients may be limited. Although we aimed to compose a balanced sample of research and clinical practice perspectives for our Delphi survey, we ultimately recruited mostly practitioners, only a small number of relatives and no patient representatives. Participating HPs were multi-professional experts with more than $60 \%$ of them being nurses. Statistical analyses displayed no significant differences between professions; however the numbers in each profession may not have been sufficient to detect such differences.

\section{Clinical Implications}

As HPs are frequently confronted with DD, a semi-standardized communication guide for dealing with DD has great potential for clinical practice. The fact that more practitioners participated in the Delphi survey than originally planned shows their great interest in the topic and its particular relevance in PC. The clinical guideline thus became a tool tailored directly towards everyday practice of people working in the field. This can include all professions directly in contact with palliative patients provided they have partaken in the mandatory training course: physicians, nurses, psychologists, social and spiritual care workers as well as volunteers. We expect the guideline in conjunction with our training program to foster multi-professional competencies across all health care structures, especially on dealing with DD in patients with serious health-related suffering due to severe illness. 


\section{Conclusions}

The major achievement of this study is the creation of the first consensus-based semi-standardized approach for (proactively) assessing and optimally responding towards DD based on literature review, patient interviews and expert consent. Besides recommendations for communication, the clinical guideline lists possible types, meanings and functions of DD as well as feasible interventions. Also HPs' self-reflection on own attitudes and emotions concerning DD as well as self-protection are taken into consideration.

\section{List Of Abbreviations}

CALM Managing cancer and living meaningfully

COREQ Consolidated criteria for reporting qualitative research

CREDES Guidelines for Conducting and reporting Delphi Studies in palliative care

DD Desire to Die

DDRS Desire for Death Rating Scale

HPs Health professionals

PC Palliative care

SAHD Schedules of Attitudes for Hastened Death

StGB National criminal code

WTHD Wish to hastened death

\section{Declarations}

Ethics approval and consent to participate

Ethical approval for this study was obtained from the Ethics Committee of the University of Cologne (\#17-265). The study is registered in the German Clinical Trials Register (DRKS00012988).

Consent for publication

Not applicable.

Availability of data and materials

The datasets generated during and/or analyzed during the current study are not publicly available, but are available from the corresponding author on reasonable request.

Competing interests

The authors declare that they have no competing interests.

Funding

The study was funded by the German Federal Ministry of Education and Research (grant number 01GY1706).

\section{Authors' contributions}

KK is principal investigator, responsible for study design, project management, recruitment, data collection, data analysis and dissemination. GF and VR are responsible for study design, recruitment, data collection, data analysis and dissemination. KB and TD 
are responsible for recruitment, data collection, data analysis and dissemination. MG, SJ, GR, KMP, HP and KS contributed to study design, data analysis and dissemination. $\mathrm{RV}$ is principal investigator and responsible for study design, project management, data analysis and dissemination. All authors read and approved the final manuscript.

All authors read and approved the final manuscript.

\section{Acknowledgements}

The authors would like to thank all interviewees and all participants of the Delphi survey for their cooperation.

\section{References}

1. Rodriguez-Prat A, Balaguer A, Booth A, et al. Understanding patients' experiences of the wish to hasten death: an updated and expanded systematic review and meta-ethnography. BMJ Open 2017; 7: e016659. DOI: 10.1136/bmjopen-2017-016659.

2. Ohnsorge K, Rehmann-Sutter C, Streeck N, et al. Wishes to die at the end of life and subjective experience of four different typical dying trajectories. A qualitative interview study. PLoS One 2019; 14: e0210784. DOI: 10.1371/journal.pone.0210784.

3. Nissim R, Gagliese $L$ and Rodin $G$. The desire for hastened death in individuals with advanced cancer: a longitudinal qualitative study. Soc Sci Med 2009; 69: 165-171. DOI: 10.1016/j.socscimed.2009.04.021.

4. Balaguer A, Monforte-Royo C, Porta-Sales J, et al. An International Consensus Definition of the Wish to Hasten Death and Its Related Factors. PLOS ONE 2016; 11: e0146184. DOI: 10.1371/journal.pone.0146184.

5. Rodríguez Prat A, Balaguer A, Crespo I, et al. Feeling like a burden to others and the wish to hasten death in patients with advanced illness: A systematic review. 2019.

6. Leitlinienprogramm Onkologie (Deutsche Krebsgesellschaft DK, AWMF). Erweiterte S3 Leitlinie Palliativmedizin für Patienten mit einer nicht heilbaren Krebserkrankung - Langversion 2.0, https://www.leitlinienprogramm-

onkologie.de/fileadmin/user_upload/Downloads/Leitlinien/Palliativmedizin/Version_2/LL_Palliativmedizin_2.0_Langversion.pdf (2019, accessed 27.08. 2019).

7. Voltz R, Galushko M, Walisko J, et al. Issues of "life" and "death" for patients receiving palliative care-comments when confronted with a research tool. Supportive Care in Cancer 2011; 19: 771-777. Research Support, Non-U.S. Gov't Validation Studies. DOI: 10.1007/s00520-010-0876-z.

8. Galushko M, Strupp J, Walisko-Waniek J, et al. Validation of the German version of the Schedule of Attitudes Toward Hastened Death (SAHD-D) with patients in palliative care. Palliative and Supportive Care 2015; 13: 713-723. DOI: $10.1017 /$ S1478951514000492.

9. Jones JM, Huggins MA, Rydall AC, et al. Symptomatic distress, hopelessness, and the desire for hastened death in hospitalized cancer patients. Journal of Psychosomatic Research 2003; 55: 411-418.

10. Lapierre S, Boyer R, Desjardins S, et al. Daily hassles, physical illness, and sleep problems in older adults with wishes to die. International Psychogeriatrics 2012; 24: 243-252. DOI: 10.1017/s1041610211001591.

11. Lindner R, Foerster R and von Renteln-Kruse W. Physical distress and relationship problems: exploring the psychosocial and intrapsychic world of suicidal geriatric patients. Z Gerontol Geriatr 2014; 47: 502-507. 2013/11/10. DOI: 10.1007/s00391-0130563-z.

12. Chochinov H, Wilson, K., Enns, M. Desire for death in the terminally ill. Am J Psychiatry 1995; 152: 1185-1191.

13. Wilson KG, Dalgleish TL, Chochinov HM, et al. Mental disorders and the desire for death in patients receiving palliative care for cancer. BMJ Supportive \& Palliative Care 2016; 6: 170. DOI: 10.1136/bmjspcare-2013-000604.

14. Udo C, Melin-Johansson C, Henoch I, et al. Surgical nurses' attitudes towards caring for patients dying of cancer - a pilot study of an educational intervention on existential issues. European Journal of Cancer Care 2014; 23: 426-440. DOI:

10.1111/ecc.12142.

15. Buecken R, Galushko M, Golla H, et al. Patients feeling severely affected by multiple sclerosis: How do patients want to communicate about end-of-life issues? Patient Education and Counseling 2012; 88: 318-324. DOI: 10.1016/j.pec.2012.03.010. 
16. Rodin G, Zimmermann, C., Rydall, A. The desire for hastened death in patients with metastatic cancer. Journal of Pain Symptom Management 2007; 33: 661-675.

17. Bellido-Perez M, Monforte-Royo C, Tomas-Sabado J, et al. Assessment of the wish to hasten death in patients with advanced disease: A systematic review of measurement instruments. Palliat Med 2017; 31: 510-525. 2017/01/27. DOl: $10.1177 / 0269216316669867$.

18. Baile WF, Buckman R, Lenzi R, et al. SPIKES-A six-step protocol for delivering bad news: application to the patient with cancer. Oncologist 2000; 5: 302-311.

19. Bumb M, Keefe J, Miller L, et al. Breaking Bad News: An Evidence-Based Review of Communication Models for Oncology Nurses. Clin J Oncol Nurs 2017; 21: 573-580. DOI: 10.1188/17.CJON.573-580.

20. Galushko M, Frerich G, Perrar K, et al. Desire for Hastened Death: How Do Professionals in Specialized Palliative Care React? Psycho-Oncology 2016; 25: 536-543.

21. Frerich G, Romotzky V, Galushko M, et al. Communication about the desire to die: Development and evaluation of a first needsoriented training concept - a pilot study. Palliative \& Supportive Care resubmitted.

22. Hudson PL, Schofield P, Kelly B, et al. Responding to desire to die statements from patients with advanced disease: recommendations for health professionals. Palliative Medicine 2006; 20: 703-710. DOI: 10.1177/0269216306071814.

23. Nursing RCo. When someone asks for your assistance to die. RCN guidance on responding to a request to hasten death. 2011.

24. Kremeike K, Galushko M, Frerich G, et al. The DEsire to Dle in Palliative care: Optimization of Management (DEDIPOM) - a study protocol. BMC Palliat Care 2018; 17: 30. 2018/02/21. DOI: 10.1186/s12904-018-0279-3.

25. Tong A, Sainsbury P and Craig J. Consolidated criteria for reporting qualitative research (COREQ): a 32-item checklist for interviews and focus groups. Int J Qual Health Care 2007; 19: 349-357. DOI: 10.1093/intqhc/mzm042.

26. Jünger S, Payne SA, Brine J, et al. Guidance on Conducting and REporting DElphi Studies (CREDES) in palliative care: Recommendations based on a methodological systematic review. Palliative Medicine 2017; 31: 684-706. DOI: $10.1177 / 0269216317690685$.

27. Payne S. Qualitative Methods of Data Collection and Analysis. In: Addington-Hall JM, Bruera E, Higginson IJ, et al. (eds) Research Methods in Palliative Care. Oxford: Oxford University Press, 2007, pp.139-161.

28. Bengtsson M. How to plan and perform a qualitative study using content analysis. NursingPlus Open 2016; 2: 8-14. DOI: 10.1016/j.npls.2016.01.001.

29. Morita T, Murata H, Hirai K, et al. Meaninglessness in Terminally III Cancer Patients: A Validation Study and Nurse Education Intervention Trial. Journal of Pain and Symptom Management 2007; 34: 160-170. DOI: https://doi.org/10.1016/j.jpainsymman.2006.10.021.

30. Vehling S, Malfitano C, Shnall J, et al. A concept map of death-related anxieties in patients with advanced cancer. BMJ Supportive \&amp;amp; Palliative Care 2017; 7: 427. DOI: 10.1136/bmjspcare-2016-001287.

31. DeCou CR and Schumann ME. On the latrogenic Risk of Assessing Suicidality: A Meta-Analysis. Suicide Life Threat Behav 2018; 48: 531-543. DOI: $10.1111 / \mathrm{sltb} .12368$.

32. Porta-Sales J, Crespo I, Monforte-Royo C, et al. The clinical evaluation of the wish to hasten death is not upsetting for advanced cancer patients: A cross-sectional study. Palliative Medicine 2019; 33: 570-577. DOI: $10.1177 / 0269216318824526$.

33. Khan L, Wong R, Li M, et al. Maintaining the will to live of patients with advanced cancer. Cancer journal (Sudbury, Mass) 2010; 16: 524-531. DOI: 10.1097/PPO.0b013e3181f45b90.

34. Deutscher Ethikrat. Zur Regelung der Suizidbeihilfe in einer offenen Gesellschaft: Deutscher Ethikrat empfiehlt gesetzliche Stärkung der Suizidprävention, https://www.ethikrat.org/fileadmin/Publikationen/Ad-hoc-Empfehlungen/deutsch/empfehlungsuizidbeihilfe.pdf (2014, accessed 28.01.2019).

35. Back AL, Arnold RM, Baile WF, et al. Approaching Difficult Communication Tasks in Oncology1. CA: A Cancer Journal for Clinicians 2005; 55: 164-177. DOI: 10.3322/canjclin.55.3.164.

36. Marschollek P, Bakowska K, Bakowski W, et al. Oncologists and Breaking Bad News-From the Informed Patients' Point of View. The Evaluation of the SPIKES Protocol Implementation. Journal of Cancer Education 2018: 1-6. DOI: 10.1007/s13187-017-13153. 
37. Bousquet G, Orri M, Winterman S, et al. Breaking Bad News in Oncology: A Metasynthesis. Journal of Clinical Oncology 2015; 33: 2437-2443. DOI: $10.1200 / J C 0.2014 .59 .6759$.

38. Stiefel F and Bourquin C. Communication in oncology: now we train - but how well?†. Annals of Oncology 2016; 27: $1660-1663$. DOI: 10.1093/annonc/mdw229.

39. Rodin G, Lo C, Mikulincer M, et al. Pathways to distress: the multiple determinants of depression, hopelessness, and the desire for hastened death in metastatic cancer patients. Soc Sci Med 2009; 68: 562-569. DOI: 10.1016/j.socscimed.2008.10.037.

40. Chan WCH. Being Aware of the Prognosis: How Does It Relate to Palliative Care Patients' Anxiety and Communication Difficulty with Family Members in the Hong Kong Chinese Context? Journal of palliative medicine 2011; 14: 997-1003. DOI: 10.1089/.jpm.2011.0099.

41. Parpa E, Tsilika E, Galanos A, et al. Depression as mediator and or moderator on the relationship between hopelessness and patients' desire for hastened death. Supportive Care in Cancer 2019. DOI: 10.1007/s00520-019-04715-2.

42. Rodin G, Lo C, Rydall A, et al. Managing Cancer and Living Meaningfully (CALM): A Randomized Controlled Trial of a Psychological Intervention for Patients With Advanced Cancer. J Clin Oncol 2018; 36: 2422-2432. DOI: 10.1200/JCO.2017.77.1097.

43. Serfaty M, King M, Nazareth I, et al. Manualised cognitive-behavioural therapy in treating depression in advanced cancer: the CanTalk RCT. Health Technol Assess 2019; 23: 1-106. DOI: 10.3310/hta23190.

44. Chochinov HM, Kristjanson LJ, Breitbart W, et al. Effect of dignity therapy on distress and end-of-life experience in terminally ill patients: a randomised controlled trial. The Lancet Oncology 2011; 12: 753-762. DOI: 10.1016/S1470-2045(11)70153-X.

45. Saracino RM, Rosenfeld B, Breitbart W, et al. Psychotherapy at the End of Life. The American Journal of Bioethics 2019; 19: 1928. DOI: $10.1080 / 15265161.2019 .1674552$.

46. Rizo-Baeza M, Mendiola-Infante SV, Sepehri A, et al. Burnout syndrome in nurses working in palliative care units: An analysis of associated factors. Journal of Nursing Management 2018; 26: 19-25. DOI: 10.1111/jonm.12506.

47. Medeiros de Oliveira S, Sousa LVD, do Socorro Vieira Gadelha M, et al. Prevention Actions of Burnout Syndrome in Nurses: An Integrating Literature Review. Clinical Practice \& Epidemiology in Mental Health 2019; 15: 64-73. DOI:

10.2174/1745017901915010064.

\section{Additional Files}

- Additional file 1: Text document (.word); Semi-structured interview guideline; Interview guideline with all questions for patient interviews

- Additional file 2: Text document (.word); Clinical guideline for addressing Desire to Die; Finalized version of the Clinical guideline after revision through patient interviews and consensus finding through Delphi process

- Additional file 3: Text document (.word); Introductory information to the Clinical guideline; complementary information on the Clinical guideline not consented in the Delphi process

\section{Tables}

Table 1. Sociodemographic data of the interviewees 


\begin{tabular}{|c|c|c|}
\hline \multicolumn{2}{|l|}{$N$} & 11 \\
\hline \multirow[t]{2}{*}{ Age } & $M, S D$ & $71.1(13.9)$ \\
\hline & Min, Max & 51,89 \\
\hline \multirow[t]{2}{*}{ Gender } & Male $(n, \%)$ & $7(63.3)$ \\
\hline & Female $(n, \%)$ & $4(36.4)$ \\
\hline First language & German $(n, \%)$ & $11(100.0)$ \\
\hline \multirow[t]{3}{*}{ Educational level } & Higher education entrance qualification (n, \%) & $5(45.5)$ \\
\hline & Higher secondary school ( $n, \%)$ & $5(45.5)$ \\
\hline & Lower secondary school ( $n, \%)$ & $1(9.1)$ \\
\hline \multirow[t]{4}{*}{ Vocational training } & Professional training $(n, \%)$ & $6(54.6)$ \\
\hline & University degree $(n, \%)$ & $2(18.2)$ \\
\hline & None $(n, \%)$ & $2(18.2)$ \\
\hline & Not specified $(n, \%)$ & $1(9.1)$ \\
\hline \multirow[t]{3}{*}{ Diagnosis } & Cancer (colon, lung, liver, breast \& abdominal, lower jaw, larynx, glioblastoma) ( $n, \%)$ & $7(63.6)$ \\
\hline & Geriatric multimorbidity ( $n, \%)$ & $2(18.2)$ \\
\hline & Chronic obstructive pulmonary disease (COPD) $(n, \%)$ & $2(18.2)$ \\
\hline \multirow[t]{4}{*}{ Care Setting } & Home care $(n, \%)$ & $4(36.4)$ \\
\hline & Residential care $(n, \%)$ & $3(27.3)$ \\
\hline & Hospice care $(n, \%)$ & $2(18.2)$ \\
\hline & In-patient care $(n, \%)$ & $2(18.2)$ \\
\hline \multirow[t]{4}{*}{ Interview Setting } & At home $(n, \%)$ & $4(36.4)$ \\
\hline & Hospice $(n, \%)$ & $3(27.3)$ \\
\hline & Residential care facility $(n, \%)$ & $2(18.2)$ \\
\hline & Hospital ( $n, \%)$ & $2(18.2)$ \\
\hline
\end{tabular}

Table 2. Sociodemographic data of the Delphi sample 


\begin{tabular}{|c|c|c|c|}
\hline \multicolumn{3}{|l|}{$N$} & 149 \\
\hline \multirow[t]{2}{*}{ Age } & \multicolumn{2}{|l|}{ Mean (Minimum, Maximum) } & $49.3(19,72)$ \\
\hline & & & $n(\%)$ \\
\hline \multirow[t]{2}{*}{ Gender } & \multicolumn{2}{|l|}{ Female } & $107(71.8)$ \\
\hline & \multicolumn{2}{|l|}{ Male } & $42(28.2)$ \\
\hline \multirow[t]{2}{*}{ Residence } & \multicolumn{2}{|l|}{ Germany } & $132(88.6)$ \\
\hline & \multicolumn{2}{|l|}{$\begin{array}{l}\text { Other countries } \\
\text { Spain } \\
\text { Canada } \\
\text { Switzerland, Norway } \\
\text { USA, Australia, El Salvador, Sweden, Portugal }\end{array}$} & $\begin{array}{l}17(11.4) \\
\text { - } \mathrm{n}=5 \\
\text { - } \mathrm{n}=3 \\
\text { - } \mathrm{n}=2 \text { each } \\
\text { - } \mathrm{n}=1 \text { each }\end{array}$ \\
\hline \multirow[t]{10}{*}{ Expertise $^{\dagger}$} & \multicolumn{2}{|l|}{ Nursing } & $91(61.1)$ \\
\hline & \multicolumn{2}{|l|}{ Physician } & $21(14.1)$ \\
\hline & \multicolumn{2}{|l|}{ Psychology and psychotherapy } & $9(6.0)$ \\
\hline & \multicolumn{2}{|l|}{ Spiritual care } & $11(7.4)$ \\
\hline & \multicolumn{2}{|l|}{ Ethics counseling } & $10(6.7)$ \\
\hline & \multicolumn{2}{|l|}{ Social work } & $1(0.7)$ \\
\hline & \multicolumn{2}{|l|}{ Relatives } & $12(8.1)$ \\
\hline & \multicolumn{2}{|l|}{ Research and science } & $20(13.4)$ \\
\hline & \multicolumn{2}{|l|}{ Non-practitioners, e.g. moral philosophers } & $13(8.7)$ \\
\hline & \multicolumn{2}{|l|}{ Other } & $17(11.4)$ \\
\hline \multicolumn{4}{|c|}{ Self-assessment } \\
\hline & & & $n(\%)$ \\
\hline \multirow[t]{15}{*}{ Experience in years } & \multirow[t]{4}{*}{ Dealing with desire to die (DD) in clinical practice } & $<1$ & $3(2.0)$ \\
\hline & & $1-9$ & $58(38.9)$ \\
\hline & & $\geq 10$ & $81(54.4)$ \\
\hline & & missing & $7(4.7)$ \\
\hline & \multirow[t]{4}{*}{ Dealing with suicidality in clinical practice } & $<1$ & $39(26.2)$ \\
\hline & & $1-9$ & $41(27.5)$ \\
\hline & & $\geq 10$ & $63(42.3)$ \\
\hline & & missing & $6(4.0)$ \\
\hline & \multirow[t]{4}{*}{ Studying DD from a theoretical perspective } & $<1$ & $58(38.9)$ \\
\hline & & $1-9$ & $61(40.9)$ \\
\hline & & $\geq 10$ & $21(14.1)$ \\
\hline & & missing & $9(6.0)$ \\
\hline & \multirow[t]{3}{*}{ Studying suicidality from a theoretical perspective } & $<1$ & $82(55.0)$ \\
\hline & & $1-9$ & $39(26.2)$ \\
\hline & & & \\
\hline
\end{tabular}




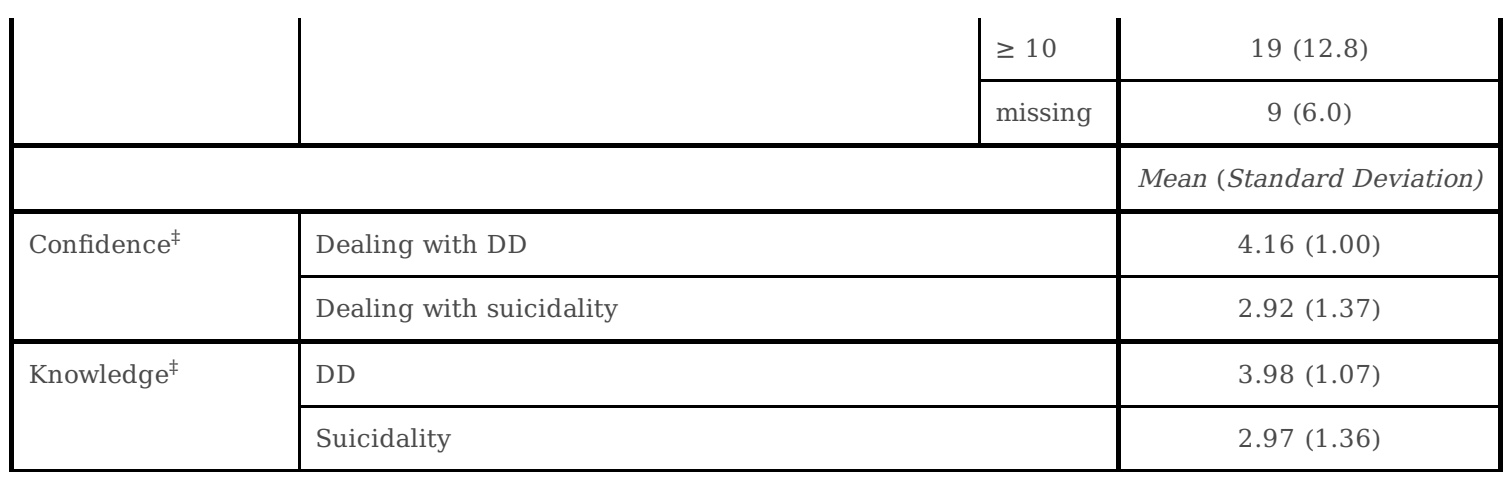

${ }^{\dagger}$ Multiple responses possible.

$\ddagger^{\prime} 0$ ' ('not confident at all') to '6' ('very confident') Likert scale adapted from Morita (2007). ${ }^{29}$ 
Table 3. Clinical guideline domains and Delphi survey importance ratings

\begin{tabular}{|c|c|c|c|c|c|c|}
\hline & \multicolumn{3}{|c|}{ Mean (Standard Deviation) } & \multicolumn{3}{|c|}{ Consensus $^{\dagger}$} \\
\hline & Round $1^{\ddagger}$ & Round $2^{\S}$ & $p$ & $\begin{array}{c}\text { Round } \\
1\end{array}$ & $\begin{array}{l}\text { Round } \\
2\end{array}$ & Increase \\
\hline \multicolumn{7}{|l|}{ A - Usage Notes } \\
\hline 1. Usage notes" & - & $\begin{array}{c}4.32 \\
(0.91)^{5}\end{array}$ & - & - & $92.6 \%$ & - \\
\hline \multicolumn{7}{|l|}{ B - Conversation Aspects } \\
\hline 2. Actively building the relationship & $\begin{array}{c}4.64 \\
(0.85)\end{array}$ & - & - & $92.6 \%$ & - & - \\
\hline 3. Proactively addressing DD & $\begin{array}{l}4.01 \\
(0.94)\end{array}$ & $\begin{array}{c}4.16 \\
(0.92)\end{array}$ & $\begin{array}{c}< \\
0.05\end{array}$ & $74.5 \%$ & $83.2 \%$ & $8.7 \%$ \\
\hline 4. Closure of discussion & $\begin{array}{c}4.62 \\
(0.74)\end{array}$ & - & - & $92.6 \%$ & - & - \\
\hline 5. After discussion & $\begin{array}{c}4.64 \\
(0.65)\end{array}$ & - & - & $94.0 \%$ & - & - \\
\hline \multicolumn{7}{|l|}{ C - Classification, Meaning and Functions } \\
\hline 6. Classification of DD & $4.26(1.0)$ & $\begin{array}{c}4.37 \\
(0.80)\end{array}$ & 0.10 & $85.2 \%$ & $90.6 \%$ & $5.4 \%$ \\
\hline 7. Background and meanings of DD & $\begin{array}{c}4.81 \\
(0.50)\end{array}$ & - & - & $97.3 \%$ & - & - \\
\hline 8. Functions of DD & $\begin{array}{c}4.31 \\
(1.07)\end{array}$ & $\begin{array}{c}4.64 \\
(0.73)\end{array}$ & $\begin{array}{c}< \\
0.01\end{array}$ & $83.9 \%$ & $95.3 \%$ & $11.4 \%$ \\
\hline \multicolumn{7}{|l|}{ D - (Self-)Reflection } \\
\hline $\begin{array}{l}\text { 9. Conscious engagement with own attitudes and } \\
\text { emotions }\end{array}$ & $\begin{array}{l}4.77 \\
(0.53)\end{array}$ & - & - & $97.3 \%$ & - & - \\
\hline 10. Self-protection & $\begin{array}{c}4.74 \\
(0.53)\end{array}$ & - & - & $96.0 \%$ & - & - \\
\hline \multicolumn{7}{|l|}{ E - Further Recommended Action } \\
\hline 11. Further recommended action & $\begin{array}{c}4.53 \\
(0.85)\end{array}$ & $\begin{array}{c}4.68 \\
(0.56)\end{array}$ & 0.07 & $87.9 \%$ & $95.3 \%$ & $7.4 \%$ \\
\hline
\end{tabular}

† Likert scale items were labeled '5' ('very important') to '1' ('very unimportant') with the option to report 'don't know' (exclusion from analysis). Consensus was assumed if participants rated domains with '4' ('quite important') or '5' ('very important'). Percentages are quotas of all participants who answered a respective question, not of the entire sample.

${ }^{\ddagger}$ For all ratings the full range of possible answers was used except for 'conscious engagement with own attitudes and emotions' $($ Min $=2$, Max $=5$ ) and 'self-protection' (Min=3, Max =5).

$\S$ For all ratings the full range of possible answers was used expect for 'further recommended action' $(\operatorname{Min}=2, \operatorname{Max}=5)$.

I Domain added after round 1. 
Table 4. Modifications of the clinical guideline based on Delphi comments

\begin{tabular}{|c|c|}
\hline Contents of comments & Implementation $^{\dagger}$ \\
\hline $\begin{array}{l}\text { free text answers across all domains pointed to the } \\
\text { need to provide general notes on proper usage of the } \\
\text { clinical guideline }\end{array}$ & $\begin{array}{l}\text { added a new domain: } \\
\text { ð 'usage notes' }\end{array}$ \\
\hline $\begin{array}{l}\text { suggestion on asking whether patients think about } \\
\text { terminating life prematurely criticized as being too } \\
\text { direct }\end{array}$ & $\begin{array}{l}\text { added a new suggestion: } \\
\text { } \text { 'Explore thoughts related to not wanting to live anymore' }\end{array}$ \\
\hline $\begin{array}{l}\text { clinical guideline seen to be at danger of provoking } \\
\text { checklist type of interrogation due to bullet point } \\
\text { setup }\end{array}$ & $\begin{array}{l}\text { changed interrogative clauses to instructions: } \\
\text { ð' Exists or existed Explore fear of death and dying ?' } \\
\text { ð' Exists or existed Explore thoughts related to terminating } \\
\text { life prematurely?' }\end{array}$ \\
\hline $\begin{array}{l}\text { complexity and changeability of desire to die (DD) in } \\
\text { palliative patients seen to run counter to } \\
\text { unambiguous classification }\end{array}$ & $\begin{array}{l}\text { added a new suggestion: } \\
\partial \text { 'In general, keep in mind: DD is complex and prone to } \\
\text { change' }\end{array}$ \\
\hline $\begin{array}{l}\text { "manipulate" in the respective function of DD seen to } \\
\text { be poor choice of words }\end{array}$ & $\begin{array}{l}\text { changed wording: } \\
\text { ð 'Attempting to manipulate influence family or health } \\
\text { professionals' }\end{array}$ \\
\hline $\begin{array}{l}\text { "attracting attention" in the respective function of DD } \\
\text { seen to be poor choice of words }\end{array}$ & $\begin{array}{l}\text { changed wording: } \\
\text { ð'Attracting Drawing. attention to oneself and one's trouble' }\end{array}$ \\
\hline $\begin{array}{l}\text { "treatment contracts" seen as bad practice, especially } \\
\text { when involving handshakes for sealing the contract } \\
\text { as it seemed to suggest "clean hands practice" }\end{array}$ & 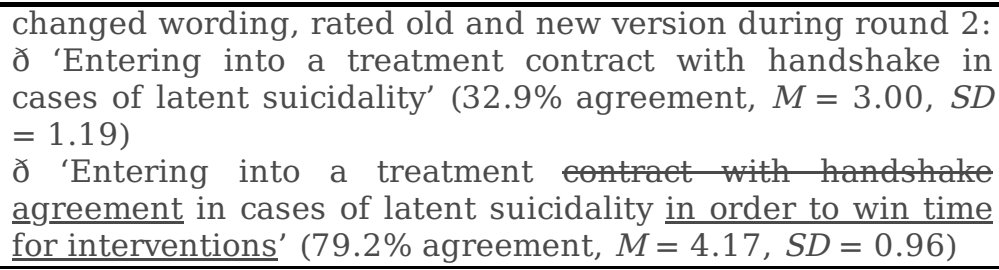 \\
\hline $\begin{array}{l}\text { suggestion on passive euthanasia seen as poorly } \\
\text { worded }\end{array}$ & $\begin{array}{l}\text { changed wording: } \\
\text { ð Letting die (passive euthanasia) as a legal option } \\
\text { (abandonment, restriction or cancellation of life sustaining. } \\
\text { and life prolonging measures).' }\end{array}$ \\
\hline $\begin{array}{lcl}\text { selection of therapeutic approaches listed } & \text { as } \\
\text { examples in respective suggestions seen as too } \\
\text { narrow }\end{array}$ & 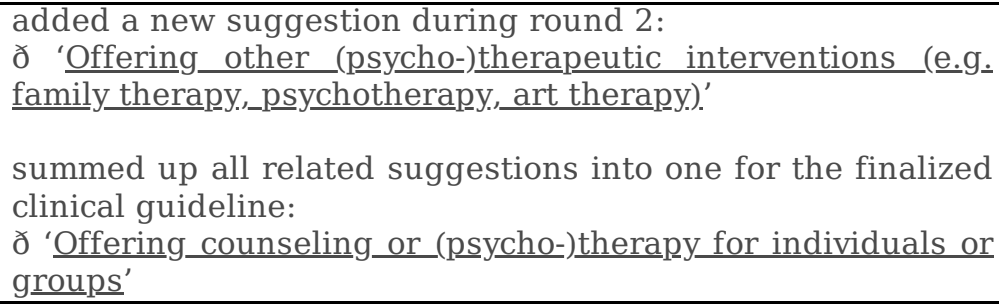 \\
\hline
\end{tabular}

${ }^{\dagger}$ plain text: same wording in round 1 and 2; strikethrough: deletions; underlined: additions

\section{Figures}




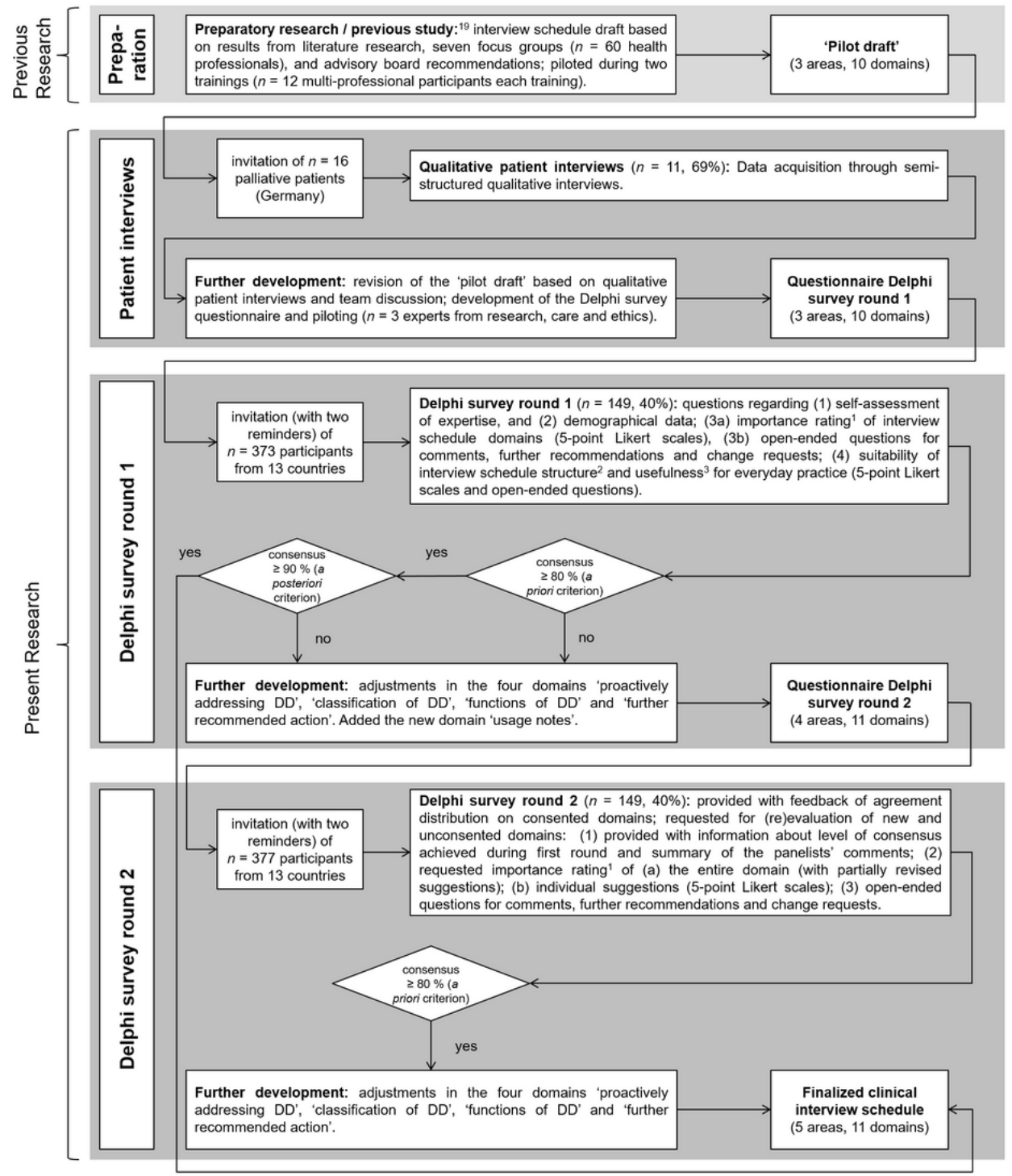

${ }^{1}$ ranging from $1=$ 'very unimportant' to $5=$ 'very important' with additional 'don't know' category excluded from analysis: ${ }^{2}$ ranging from $1=$ ' not suitable at all' to $5=$ 'very suitable' with additional 'don't know' category excluded from analysis; ${ }^{3}$ ranging from $1=$ 'not useful at all' to $5=$ 'very useful' with additional 'don't know' category excluded from analysis.

\section{Figure 1}

Flowchart of the interview schedule development process

\section{Supplementary Files}

This is a list of supplementary files associated with this preprint. Click to download.

- AdditionalFile2ClinicalguidelineforaddressingDD.docx

- AdditionalFile3Introductoryinformation.docx

- AdditionalFile1 InterviewGuideline.docx 